\title{
El Jefe de Estudios y la Comisión de Docencia ante los cambios en la Formación Especializada introducidos por el Real Decreto 183/2008
}

\author{
José Saura Llamas ${ }^{\mathrm{a}}$, José Galcerá Tomás ${ }^{\mathrm{b}}$, Carmen Botella Martínez ${ }^{\mathrm{c}}$ \\ y Miembros del Foro de Jefes de Estudios de la Región de Murcia ${ }^{d}$
}

aTécnico de Salud de la Unidad Docente de Medicina Familiar y Comunitaria de Murcia.

bJefe de Estudios de Formación Especializada, Hospital Universitario "Virgen de la Arrixaca", Murcia.

'Técnica de Apoyo Docente, Hospital Universitario "Virgen de la Arrixaca", Murcia.

'Fernando Navarro Mateu, Iñigo Anza Aguirrezabala, Andrés Conesa Hernández, Faustino Herrero Huerta, Jacinto Fernández Pardo, Julio Fontcuberta Martínez, Antonio Martínez Pastor, Magdalena Molina Oller y Andrés Carrillo Alcaraz.

Correspondencia: José Saura Llamas. E-mail: j.saurall@ gmail.com.

Recibido el 20 de noviembre de 2010.

Aceptado para su publicación el 28 de febrero de 2011.

\section{RESUMEN}

El Real Decreto 183/2008 sobre Formación Médica Especializada en Ciencias de la Salud ha supuesto un cambio sustancial de esta formación en nuestro país. Desde su publicación las Unidades Docentes (UU DD) han comenzado un proceso de adaptación a este decreto, proceso que es complejo y que por los múltiples e importantes cambios que introduce requiere mucho esfuerzo por parte de los docentes.

Este viaje presenta dificultades añadidas por el incumplimiento de casi todas las instituciones competentes de elaborar la normativa que desarrolle la mayoría de sus contenidos, y por lo tanto las UU DD carecen de las guías y orientaciones al respecto.

Como iniciativa de la Comisión de Docencia de Formación Especializada del Hospital Universitario Virgen de la Arrixaca, se presenta el resultado del trabajo en grupo de los asistentes (informadores clave) al taller de Actualización en la Normativa sobre las Figuras Docentes en la Formación Sanitaria Especializada celebrado en Murcia, febrero de 2010. En este caso se centra en las propuestas elaboradas sobre las figura del Jefe de Estudios de las Unidades Docentes y la Comisión de Docencia, con la intención de aportar ayuda para los jefes de estudios y los docentes en esta adaptación.

Palabras clave. Educación de Postgrado, Normas Técnicas.

\section{ABSTRACT}

The Head of Studies and the Education Committee face the changes in Specialised Training introduced in the Royal Decree 183/2008

The Spanish Royal Decree 183/2008 on Specialised Medical Training in Health Sciences has meant a substantial change to this kind of training in Spain. Since its publication, the Teaching Units (TUs) have started a process of adaptation to this decree, a process that is complex and that requires much effort in view of the many significant changes.

The process is complicated even more because the competent institutions have failed to develop regulation on most of the Decree's contents, leaving the TUs with no guidelines.

As an initiative of the Education Committee on Specialised Training of the Teaching Hospital 'Virgen de la Arrixaca' we present the results of the teamwork (key informers) in a workshop on the Update of the Regulation on Educators in Specialised Health Training that took place in Murcia in February 2010. We focus on the proposals developed for the Head of Studies of the Teaching Units and the Education Committee, with the aim of providing support to the directors of studies and teachers in this adaptation.

Key words. Education, Graduate, Technical Standards.

Del Taller de Actualización en la Normativa sobre las Figuras Deocentes en la Formación Sanitaria Especializada, celebrado en

Murcia, febrero de 2010.

\section{INTRODUCCIÓN}

El sistema MIR de formación médica especializada se inició en nuestro país en los años 60 de una manera minoritaria, y se extendió dentro del Sistema Nacional de Salud tras varias experiencias piloto de éxito. El conjunto de la formación MIR adquirió carta de naturaleza con la promulgación del RD $127 / 1984^{1}$, en el que se estructuraba y regulaba esta formación, aunque de una manera muy general. 
En la orden de $1995^{2}$ fueron reconocidos los diferentes aspectos y mencionadas por primera vez las diferentes figuras participantes en la formación especializada.

Paradójicamente y a pesar de la existencia de esta y otra abundante normativa, la formación MIR era globalmente una formación poco regulada y escasamente normalizada, con una muy pobre definición del proceso formativo, con una ausencia muy llamativa de datos e información sobre el seguimiento y la evaluación del proceso formativo, y sobre todo con una falta de definición y descripción detallada de las funciones de las distintas figuras docentes.

Esta situación, añadida a la necesaria adaptación al Espacio Europeo de Formación Superior, ha hecho que recientemente hayan aparecido nuevas normas, entre las que destaca el RD 183/20083', que han introducido cambios sustanciales en el proceso de Evaluación, el control de calidad de la formación, en las funciones de algunas de las figuras docentes y especialmente en la ampliación de las competencias del papel del tutor ${ }^{3}$, descritas con mucho detalle, así como las funciones del Jefe de Estudios ${ }^{3}$, compartidas o no con la Comisión de Docencia correspondiente.

El artículo 13 del citado RD 183/2008³, titulado "otras figuras docentes", recoge la posibilidad de que las comunidades autónomas, según sus características y criterios organizativos propios, puedan crear otras figuras docentes con la finalidad de amparar colaboraciones significativas en la formación especializada, objetivos de investigación, desarrollo de módulos genéricos o específicos de los programas o cualesquiera otras actividades docentes de interés. Aunque no menciona expresamente a algunas figuras que ya existen dentro del sistema MIR como son el Técnico de Salud Pública o Técnico de Apoyo Docente y el Jefe de Residentes.

Pero la actual normativa ha dejado muchos aspectos, con gran repercusión práctica en el desempeño de los roles docentes, a la publicación de nuevas normas por las diferentes comunidades autónomas (CCAA) de acuerdo con las disposiciones de la Ley de Ordenación de Profesiones Sanitarias ${ }^{4}$. Sin embargo, el plazo contemplado en el RD 183/2008 para el desarrollo de dicha normativa autonómica finalizó en febrero de $2009^{3}$.

Aunque este incumplimiento ha supuesto un freno a la posible implantación al desarrollo de la normativa y a la mejora del proceso formativo de los MIR, como aspecto positivo ha permitido disponer de un tiempo de reflexión para que los responsables de las CCAA puedan tener en cuenta la opinión de los principales actores de la formación especializada con relación al desempeño de los roles de las distintas figuras docentes. Curiosamente, y a pesar de que estos colectivos de docentes (sobre todo los tutores) tienen una gran experiencia en el desempeño de estas labores, hasta ahora prácticamente no han sido consultados para elaborar estas normas.

\section{MATERIAL Y MÉTODOS}

Ante esta situación la Comisión de Docencia Especializada del Hospital Universitario "Virgen de la Arrixaca", a través de su Jefe de Estudios, y con la intención de recoger las opiniones de los docentes que vienen desempeñando dichas tareas formativas, puso en marcha una investigación cualitativa durante el mes de Febrero de 2010, bajo la forma de un Taller denominado de «actualización de la normativa sobre las figuras docentes en la Formación Sanitaria Especializada».

Esta actividad ha constado de 3 sesiones de 5 horas, desarrolladas en tres días en horario de tarde, y en cada una de ellas se ha tratado una de las siguientes 3 figuras docentes: el Jefe de Residentes, el Técnico Docente y el Jefe de Estudios (y la Comisión de Docencia). En ella han participado como informadores clave: jefes de estudios de todas las Unidades Docentes de Formación Especializada, técnicos de salud y técnicos docentes, jefes de residentes, tutores y otros docentes de la CA de la Región de Murcia, con 43 inscritos y una participación media de 30 personas.

La actividad se desarrollaba en 2 partes, realizándose en la primera una exposición por varios docentes expertos en cada una de las tres figuras docentes objeto del taller, de $11 \frac{1}{2}$ hora de duración, y exponiéndose los orígenes, la situación actual y propuestas para su desarrollo de cada una de ellas. En la segunda parte, de unas 3 horas de duración, se desarrollaba un trabajo en grupo, con un moderador que además recogía los resultados, bajo la doble forma de la tormenta de ideas y el grupo nominal, elaborándose al finalizar cada sesión un documento de consenso con las conclusiones y propuestas concretas de estos docentes sobre a la definición, funciones y otras características deseables para estas figuras docentes. Los resultados referidos al Jefe de Estudios y a la Comisión de Docencia son los que se exponen en este artículo. 


\begin{tabular}{|c|c|c|}
\hline \multicolumn{3}{|c|}{ FUNCIONES RELACIONADAS CON EL CENTROIAREA } \\
\hline FUNCIONES & Comisión Docencia & Jefatura Estudios \\
\hline Presidencia & & $\begin{array}{l}\text { Asumir la presidencia de la comisión de docencia, } \\
\text { dirimiendo con su voto los empates que se produzcan } \\
\text { en la adopción de acuerdos }\end{array}$ \\
\hline Representación & & $\begin{array}{l}\text { Asumir la representación de la comisión de docencia } \\
\text { formando parte, en los términos que establezcan las } \\
\text { comunidades autónomas, de los órganos de dirección } \\
\text { de los correspondientes centros y servicios sanitarios, } \\
\text { con el fin de asegurar y garantizar la incardinación } \\
\text { de la docencia en la actividad asistencial ordinaria, } \\
\text { continuada y de urgencias de dichos centros }\end{array}$ \\
\hline Capacidad docente & $\begin{array}{l}\text { Informar, al menos anualmente, a los } \\
\text { correspondientes órganos de dirección sobre la } \\
\text { capacidad docente del centro o unidad }\end{array}$ & \\
\hline $\begin{array}{l}\text { Coordinación } \\
\text { docencia- } \\
\text { asistencia }\end{array}$ & $\begin{array}{l}\text { Facilitar la adecuada coordinación docente entre } \\
\text { niveles asistenciales. }\end{array}$ & $\begin{array}{l}\text { Actuar como interlocutor entre los responsables } \\
\text { asistenciales y docentes con la finalidad de garantizar } \\
\text { una adecuada coordinación entre los mismos. }\end{array}$ \\
\hline $\begin{array}{l}\text { Gestión de calidad } \\
\text { docente }\end{array}$ & $\begin{array}{l}\text { Aprobar el plan de gestión de calidad docente } \\
\text { del centro o unidad docente, supervisando su } \\
\text { cumplimiento, a cuyos efectos les será facilitada } \\
\text { cuanta información sea necesaria por los } \\
\text { responsables de las unidades asistenciales y por los } \\
\text { correspondientes órganos de dirección y gestión. }\end{array}$ & $\begin{array}{l}\text { Supervisar el plan de gestión de calidad docente del } \\
\text { centro o unidad. }\end{array}$ \\
\hline
\end{tabular}

Tabla 1. Funciones correspondientes a la Comisión de Docencia y/o al Jefe de Estudios relacionadas con el centro o area (RD 183/2008)

\section{LA FIGURA DEL JEFE DE ESTUDIOS Y LA COMISIÓN DE DOCENCIA}

\section{Orígenes y justificación}

La figura del Jefe de Estudios queda ampliamente definida en el RD183 de $2008^{3}$, que recoge, concreta y amplia todas las funciones y características previamente descritas en la Orden 22 de junio de 1995², Orden 3 de marzo 20056, RD 1146 de $2006^{7}$ y Orden 22 de febrero de $2005^{8}$. Asimismo, en el mencionado decreto se describen y regulan la composición y funciones de las Comisiones de Docencia.

Sin embargo, tanto en la legislación previa como en su actualización no se establece un límite claro entre las funciones de Jefe de Estudios y las de la Comisión de Docencia, solapando en muchos aspectos tareas. El solapamiento de las funciones suele derivar en la práctica en que muchas recaigan y desborden la capacidad de la Jefatura de Estudios. Por ello, es un punto crucial en el momento actual delimitar cuáles son las competencias de una y/u otra figura.

\section{Ordenación y separación de las funciones que corresponden a la Comisión de Docencia y al Jefe de Estudios}

Se elaboró, previamente a la realización del taller, una tabla revisando el mencionado RD y la orden posterior de 2008, tratando de identificar, ordenar y asignar cada de una de las funciones según correspondieran a la Comisión de Docencia y/o al Jefe de Estudios (tablas 1 a 4).

En este documento y por consenso de los asistentes al taller se pretende recoger las funciones de ambas figuras docentes y proponer qué funciones serían "exclusivas" del Jefe de Estudios, y cuáles de ellas pueden ser delegadas en otros miembros o figuras de la Comisión de Docencia.

De acuerdo con los mencionados puntos relativos a las funciones de la Comisión de Docencia y 
FUNCIONES RELACIONADAS CON LOS RESIDENTES

\begin{tabular}{|c|c|c|}
\hline FUNCIONES & Comisión Docencia & Jefatura Estudios \\
\hline $\begin{array}{l}\text { Comités de } \\
\text { evaluación }\end{array}$ & Constitución y designación de facultativo para el comité. & $\begin{array}{l}\text { Presidir, según prevé la legislación vigente, } \\
\text { los correspondientes comités de evaluación } \\
\text { anual, dirimiendo con su voto los empates que } \\
\text { pudieran producirse. }\end{array}$ \\
\hline $\begin{array}{l}\text { Evaluaciones } \\
\text { formativas }\end{array}$ & $\begin{array}{l}\text { Facilitar las herramientas para que pueda llevarse a cabo la } \\
\text { evaluación formativa en el centro. Supervisar su realización. }\end{array}$ & \\
\hline $\begin{array}{l}\text { Evaluaciones } \\
\text { finales y anuales }\end{array}$ & $\begin{array}{l}\text { Remitir al Registro Nacional de Especialistas en Formación, } \\
\text { a través de su presidente, las evaluaciones finales y } \\
\text { anuales, así como los resultados de sus revisiones y los } \\
\text { períodos de recuperación que en su caso correspondan, en } \\
\text { los términos previstos en la legislación vigente }\end{array}$ & $\begin{array}{l}\text { Garantizar la correcta remisión, en tiempo } \\
\text { y forma, de las evaluaciones y demás } \\
\text { documentación que se deba trasladar al } \\
\text { Registro de Especialistas en Formación del } \\
\text { Ministerio de Sanidad y Consumo. }\end{array}$ \\
\hline $\begin{array}{l}\text { Guía o itinerario } \\
\text { formativo tipo }\end{array}$ & $\begin{array}{l}\text { Aprobar, a propuesta de los correspondientes tutores, } \\
\text { una guía o itinerario formativo tipo de cada una de las } \\
\text { especialidades que se formen en su ámbito. Dicha guía, que } \\
\text { garantizará el cumplimiento de los objetivos y contenidos } \\
\text { del programa oficial de la especialidad, se adaptará a las } \\
\text { características específicas de cada centro o unidad. }\end{array}$ & \\
\hline $\begin{array}{l}\text { Plan individual de } \\
\text { formación }\end{array}$ & $\begin{array}{l}\text { Garantizar que cada uno de los residentes de las } \\
\text { especialidades que se formen en su centro o unidad, } \\
\text { cuenten con el correspondiente plan individual de } \\
\text { formación, verificando en colaboración con los tutores de } \\
\text { la especialidad de que se trate, su adecuación a la guía } \\
\text { formativa o itinerario tipo antes citado. }\end{array}$ & \\
\hline $\begin{array}{l}\text { Supervisión de los } \\
\text { residentes }\end{array}$ & $\begin{array}{l}\text { Elaborar el protocolo de supervisión de los residentes en los } \\
\text { términos establecidos en la legislación vigente. }\end{array}$ & $\begin{array}{l}\text { Consensuar y suscribir con los correspondientes } \\
\text { órganos de dirección del centro en } \\
\text { representación de la comisión de docencia, el } \\
\text { protocolo de supervisión de los residentes según } \\
\text { la legislación vigente }\end{array}$ \\
\hline $\begin{array}{l}\text { Participación de } \\
\text { los residentes en } \\
\text { cursos }\end{array}$ & $\begin{array}{l}\text { Aprobar y fomentar la participación de los residentes en } \\
\text { cursos, congresos, seminarios o reuniones científicas, } \\
\text { relacionados con el programa, previo informe de la unidad } \\
\text { de apoyo a la formación/investigación que en cada caso } \\
\text { corresponda, oído el tutor y el responsable de la unidad } \\
\text { asistencial de que se trate. }\end{array}$ & \\
\hline
\end{tabular}

Tabla 2. Funciones correspondientes a la Comisión de Docencia y/o al Jefe de Estudios relacionadas con los residentes (RD 183/2008)

de la Jefatura de Estudios, nuestra propuesta es identificar como funciones "ejecutivas" aquellas que corresponden principalmente o son responsabilidad directa de cada una de las figuras comentadas.

\section{Funciones ejecutivas de la Comisión de Docencia: organizar y gestionar las actividades de formación}

- Aprobar y supervisar los programas de formación.
- Supervisar la aplicación práctica de la formación y su integración con la actividad asistencial del centro.

- Elaborar, aprobar y supervisar del plan de calidad docente.

- Elaborar el protocolo de supervisión de los residentes.

- Estudiar la capacidad formativa de las unidades docentes.

- Acreditar y supervisar tutores. 


\section{FUNCIONES RELACIONADAS CON LOS TUTORES}

\begin{tabular}{l|l|l}
\hline FUNCIONES & \multicolumn{1}{|c}{ Comisión Docencia } & Jefatura Estudios \\
\hline $\begin{array}{l}\text { Formación continuada } \\
\text { de los tutores }\end{array}$ & $\begin{array}{l}\text { Facilitar la formación continuada de los tutores en } \\
\text { metodologías docentes y otros aspectos relacionados } \\
\text { con los programas formativos }\end{array}$ & \\
\hline $\begin{array}{l}\text { Acreditación y } \\
\text { reacreditación de } \\
\text { tutores }\end{array}$ & $\begin{array}{l}\text { Participar en la acreditación y reacreditación de tutores } \\
\text { en los términos que establezca cada comunidad } \\
\text { autónoma }\end{array}$ & \\
\hline
\end{tabular}

Tabla 3. Funciones correspondientes a la Comisión de Docencia y/o al Jefe de Estudios relacionadas con el centro o area (RD 183/2008)

\section{FUNCIONES RELACIONADAS CON LOS RECURSOS}

\begin{tabular}{l|l|l}
\hline FUNCIONES & \multicolumn{1}{|c}{ Comisión Docencia } & Jefatura Estudios \\
\hline $\begin{array}{l}\text { Recursos estructurales } \\
\text { actividades docentes }\end{array}$ & $\begin{array}{l}\text { Procurar que en los dispositivos del centro } \\
\text { o unidad se den las condiciones necesarias } \\
\text { para impartir una adecuada formación a los } \\
\text { residentes, así como para llevar a cabo la } \\
\text { evaluación formativa de las actividades de } \\
\text { los mismos, procediendo a la revisión de } \\
\text { las evaluaciones anuales en los términos } \\
\text { previstos en la legislación vigente. }\end{array}$ & \\
\hline $\begin{array}{l}\text { Medios materiales } \\
\text { y personales para } \\
\text { Tutores }\end{array}$ & $\begin{array}{l}\text { Proponer a los correspondientes órganos } \\
\text { de dirección que adopten las medidas } \\
\text { necesarias para que se dote a las } \\
\text { comisiones de docencia y a los tutores } \\
\text { de los medios materiales y personales } \\
\text { que sean necesarios para la adecuada } \\
\text { realización de sus funciones }\end{array}$ & \\
\hline $\begin{array}{l}\text { Tablón oficial de } \\
\text { anuncios de la } \\
\text { Comisión }\end{array}$ & $\begin{array}{l}\text { Comunicar por escrito a los residentes el } \\
\text { lugar donde se ubicará el tablón/es oficial/ } \\
\text { es de anuncios de la Comisión en el que } \\
\text { se insertarán los avisos y resoluciones de } \\
\text { la misma }\end{array}$ & $\begin{array}{l}\text { Ordenar la inserción en el tablón de anuncios de los } \\
\text { avisos y resoluciones de la comisión de docencia } \\
\text { que requieran publicación insertando la diligencia } \\
\text { relativa a la fecha de publicación que en cada caso } \\
\text { corresponda. }\end{array}$ \\
\hline
\end{tabular}

Tabla 4. Funciones correspondientes a la Comisión de Docencia y/o al Jefe de Estudios relacionadas con el centro o area (RD 183/2008)

- Facilitar su formación continuada.

- Aprobar la participación de residentes en congresos y cursos.

- Aprobar rotaciones externas/estancias formativas.

- Relaciones con organismos externos.

\section{Funciones Ejecutivas del Jefe de Estudios}

- Presidencia de la comisión de docencia.

- Dirigir y coordinar la actividad de los tutores.

- Representar a la comisión de docencia.

- Actuar de interlocutor entre asistenciales y docentes.

- Pactar con la dirección asistencial el protocolo de supervisión de los residentes.

- Supervisar el plan de calidad.

- Presidir comités de evaluación.

- Promover y definir líneas de investigación docente.

- Relaciones con la administración.

- Gestionar los recursos de la comisión de docencia. 


\section{Cronograma de las funciones del jefe de estudios}

Con el fin de racionalizar y dar un horizonte temporal de las diferentes funciones se propone el siguiente cronograma:

Gestiones diarias:

- Autorizaciones a congresos, cursos, etc.

- Gestiones de rotaciones externas (residentes de su centro o que vienen a su centro).

- Certificaciones varias.

- Reuniones con jefes o tutores o residentes.

Gestiones semanales:

- Reuniones con tutores, con jefes y residentes para entrevistas y consenso acreditaciones tutores.

Gestiones mensuales:

- Reuniones con la Comisión de Docencia: rotaciones externas (información), pero sobre todo internas (acuerdos), urgencias y otros servicios, acreditaciones internas de tutores y adecuaciones de programas docentes.

- Seguimiento del desarrollo de Proyectos Docentes.

Gestiones anuales:

- Seguimiento de la calidad docente (y actuaciones subsiguientes).

- Encuestas a residentes (y actuaciones subsiguientes).

- Evaluaciones (con sus comités de evaluación).

- Organización anual de las recepciones y despedidas.

- Organización anual de concursos de becas y premios.

- Organización anual de cursos de metodología docente.

- Organización anual de la formación complementaria.

Otras gestiones variables:

- Seguimiento-atención periódica de las auditorías docentes.

- Atenciones externas (redes autonómica y nacional, SAS, CS).

\section{CONCLUSIONES Y PROPUESTAS}

Las funciones de la Comisión de Docencia y Jefe de Estudios pueden entrar en conflicto o solaparse, especialmente, en los siguientes aspectos:

1. Coordinación docente entre niveles asistenciales. Corresponde al Jefe de Estudios ser el interlocutor entre los responsables asistenciales y docentes. No obstante, la
Comisión de Docencia podrá designar uno o más representes para que esta coordinación se lleve a cabo de la forma más flexible y adecuada según la complejidad de las Unidades Docentes y dispositivos asistenciales.

2. Coordinación entre enseñanzas de grado y posgrado. La Comisión de Docencia propondrá y resolverá al representante que ejercerá como interlocutor entre la Universidad y el Centro, y se integrará como tal en el organismo pertinente, que suele ser la Comisión mixta HospitalUniversidad y/o Universidad Sistema Murciano de Salud.

3. Gestión de calidad docente. Por su complejidad, novedad, y especificidad, este es uno de los aspectos que más preocupan a los Jefes de Estudios y a las Figuras Docentes. Durante el Taller se trabajaron y discutieron las distintas posibilidades para la elaboración y puesta en marcha de los Planes de Gestión de la Calidad Docente (PGDC). Se consensúo en establecer las siguientes responsabilidades: elaboración por el Jefe de Estudios, Técnico Docente, y asesoría de la Comisión de Calidad; el soporte técnico corresponde a los técnicos acreditados en Calidad; aprobación por la Comisión de Docencia; cronograma de aplicación explícito en el PGCD, siendo éste uno de sus epígrafes; aplicación: corresponde a la Comisión de Docencia, a través del Jefe de Estudios y/o el Técnico Docente, el desarrollo del PGCD; su implantación y difusión entre los responsables asistenciales y docentes mediante un calendario de reuniones al inicio y semestrales tras el mismo; se revisarán por la Comisión de Docencia los indicadores en los plazos determinados por el PGCD y se trasladarán al PGC del centro y a la Alta Dirección.

4. Auditorías Docentes. Propuesta por parte de la Comisión de Docencia por iniciativa propia o tras propuesta por alguna figura o Unidad Docente o asistencial (auditorías internas), o por los clientes (auditorias externas de segunda parte) o bien por organismos independientes (auditorías externas de terceras partes). Contacto inicial: Auditores con Jefe de Estudios. Preparación de la Auditoria: Jefe de Estudios, Técnicos docentes, Jefes de Residentes en colaboración con Jefe y Tutores de los servicios auditados. Desarrollo de la Auditoría: entrevistas con Jefe, Tutores y Residentes de los Servicios Auditados, con Jefe de Estudios, 
con la Alta Dirección, y recopilación y verificación de evidencias demandadas en la auditoria. Planes de mejora: elaboración del Plan de Mejora por parte del Jefe de estudios, Técnico Docente y Responsables del Servicio auditado. Seguimiento del Plan de mejora: Jefe de Estudios y Técnico Docente. Envío de la Documentación del Plan de mejora: Jefe de Estudios.

5. Supervisión de los residentes. Responsable de elaboración: cada Unidad Docente o Asistencial. Criterios: nivel de competencia, nivel de responsabilidad y grado de supervisión. Aprobación: Comisión de Docencia.

6. Acreditación y reacreditación de tutores. No existen, de momento, criterios de acreditación y reacreditación, estando pendientes de ser dados como normativa por parte del organismo pertinente. En la discusión se coincide en la importancia crucial de este aspecto.

\section{BIBLIOGRAFÍA}

1. Real Decreto $127 / 1984$, de 11 de enero, por el que se regula la Formación Médica Especializada y la obtención del Título de Médico Especialista. BOE 11 de Enero 1984.

2. Orden de 22 de junio de 1995 , por la que se regulan las Comisiones de Docencia y los sistemas de evaluación de la formación de los médicos y farmacéuticos especialistas. Ministerio de la Presidencia. BOE 30 de junio 1995.

3. Real Decreto $183 / 2008$, de 8 de febrero, por el que se determinan y clasifican las especialidades en Ciencias de la Salud y se desarrollan determinados aspectos del sistema de formación sanitaria especializada. Ministerio de la Presidencia. BOE 21 de Febrero 2008.

4. Ley de ordenación de las profesiones sanitarias. BOE 21 de noviembre 2003.

5. Orden de 19 de diciembre de 1983, por la que se regula el desarrollo de la formación en atención primaria de Salud de la Especialidad de Medicina Familiar y Comunitaria. BOE 22 de diciembre 1983.

6. Orden SCO/1198/2005, de 3 de marzo, por la que se aprueba y publica el programa formativo de la especialidad de Medicina Familiar y Comunitaria. BOE 3 de mayo de 2005.

7. Real Decreto $1146 / 2006$, de 6 de Octubre, por el que se regula la relación laboral especial de residencia para la formación de especialidades en ciencias de la salud. BOE 7 de Octubre de 2006.

8. Orden SCO/581/2008, de 22 de febrero, por la que se publica el Acuerdo de la Comisión de Recursos Humanos del Sistema Nacional de Salud, por la que se fijan los criterios generales relativos a la composición y funciones de las comisiones de docencia, a la figura del jefe de estudios de formación especializada y al nombramiento del tutor. BOE 5 de marzo 2008. 